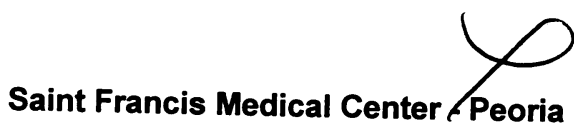

\title{
Guidelines for Specialist Training in Accident and Emergency Medicine
}

Prepared by the Board of the Faculty of Accident and Emergency Medicine 


\section{Guidelines for Specialist Training in Accident and Emergency Medicine}

Prepared by the Board of the Faculty of Accident and Emergency Medicine

\section{Contents}

Introduction 1

Section 1 Regulations relating to the Specialty Examination 1

Section 2 Curriculum for Higher Specialist Training 2

Section 3 Proforma for assessment 6 
This document has been developed by the Faculty and the Specialist Advisory Committee in A\&E Medicine to provide guidance for both junior doctors who are considering a career in the specialty and trainees who have embarked upon higher specialist training. It should also be of value to trainers and all those responsible either directly or indirectly for providing learning opportunities for those aspiring to consultant status in accident and emergency medicine.

It is always difficult when preparing curricula to strike the appropriate balance between bland general statements and detailed lists which are inevitably incomplete. I hope that our compromise is successful in answering more questions than it raises.

The format of the examination has been determined after careful assessment of our training objectives. We are grateful to the Joint Committee on Intercollegiate Examinations and to our educational advisers for their help in developing a form of assessment which combines criteria based objectivity with the opportunity to take a more reflective approach to the examination of experienced and senior trainees.

The Training Record attempts to bring together information which will be of value to trainee and trainer as they progress together through the training programme.

This first attempt to combine these three documents is the product of much hard work by many groups within the specialty. I hope that you find the result of value and that you will contribute to the second edition by sending any constructive comments to the Registrar of the Faculty.

DW YATES

Dean

January 1996 


\section{GUIDELINES FOR}

SPECIALIST TRAINING

IN ACCIDENT AND EMERGENCY

MEDICINE

\section{INTRODUCTION}

This booklet is issued as guidance and will be kept under review by the Specialist Advisory Committee and the Faculty of Accident and Emergency Medicine. It comprises the regulations relating to the specialty examination in A\&E medicine, a curriculum for higher specialist training, and a copy of the training record.

The curriculum is designed to give trainees and trainers in
A\&E medicine guidelines for clinical experience in a wide variety of emergencies. Its content is not meant to be exhaustive but to highlight areas of clinical knowledge, skills and attitudes relevant to higher specialist training in $A \& E$ medicine. Training should emphasise proficiency in resuscitation, assessment, initial management, and appropriate referral of patients as well as responsibilities for organisational and administrative aspects of an $A \& E$ service.

\section{SECTION 1}

\section{Regulations relating to the Specialty Examination in Accident and Emergency Medicine}

\subsection{Criteria for Eligibility}

The criteria for eligibility to sit the Fellowship examination are as follows:

(i) The candidate must have been medically qualified for at least 7 years.

(ii) The candidate must:

- have held a Fellowship of one of the Royal Colleges of Surgeons of Great Britain or Ireland for at least 3 years;

OR

- have held the MRCP(UK) or FRCA or equivalent for at least 3 years;

OR

- have acquired qualifications acceptable to the Faculty Board in lieu of these requirements.

(iii) The candidate must have completed 3 years in a programme of specialty training acceptable to the Examination Committee of the Faculty of Accident and Emergency Medicine. At least one year of this training must have been in an approved Higher Specialist Training post in Great Britain or Ireland or in an equivalent post overseas approved by the Faculty Examination Committee. Part time trainees must have completed the full time equivalent of these periods. Candidates training in Great Britain or Ireland must be enrolled with the Specialist Advisory Committee in Accident and Emergency Medicine.
In case of dispute as to eligibility the decision of the Faculty's Examination Committee shall be final.

\subsection{Application}

1.2.1 Candidates will be required to submit notice of intention to take this examination eight weeks before the date of the examination.

1.2.2 Application for admission to the examination must be accompanied by all the required documentation and the full amount of the fee payable for the examination. Candidates are required to submit a precis of their Training Record at the time of application. The original Training Record should be brought to the examination by hand. This should be loosely bound and conform to the format laid down by the Examination Committee.

1.2.3 Candidates withdrawing from the examination must do so in writing. The whole entrance fee may be returned when written notice is received before the closing date for receipt of applications. Half of the entrance fee may be refunded or transferred to a future examination when written evidence is received not less than 21 days before the start of the examination. After that date no refund of the fee will be made to candidates who withdraw or fail to attend. 


\section{$1.3 \quad$ Format}

There are four components to the examination:

(1) A discussion of the contents of the candidate's Training Record will occupy 30 minutes. Particular reference will be made to the sections on clinical topic reviews, research, and A\&E management.

(2) The candidate will be given a recently published research or clinical paper to study for one hour before a 30 minute discussion of its content. The paper will be chosen for its general application to A\&E clinical practice.

(3) Forty five minutes will be devoted to A\&E management. Part of this will be a structured discussion of a developing clinical scenario, with the remaining time devoted to a variety of clinical and administrative topics relevant to the Specialty.

(4) An objective structured clinical examination will occupy the final 45 minutes of the examination. This will use clinically based material such as slides, $x$ rays, ECG, laboratory data, instruments, equipment, and any other material considered to be appropriate.

\subsection{Guide to the Scope of the Examination}

The examination will be broadly based and offer an opportunity for candidates to demonstrate the breadth of their knowledge of accident and emergency medicine and special areas of interest. Proficiency will be expected in the clinical management of all conditions which can reasonably be expected to present to an $A \& E$ department. The candidate will be expected to demonstrate knowledge of information resources relevant to emergency patient care both within and without the hospital setting. An understanding of research methodology will be expected, and the candidate should be able to assess the soundness and clinical relevance of research work. The candidate must be able to demonstrate good communication skills and a knowledge of educational principles applicable to $\mathrm{A} \& \mathrm{E}$ medicine at both undergraduate and postgraduate levels. The candidate will also be expected to demonstrate knowledge of the management skills required to run an efficient and effective accident and emergency service.

\section{$1.5 \quad$ Addendum}

Candidates who have completed periods of training before receiving the Regulations for the Examination and before receiving the Training Record will not be expected to complete their Training Record for such periods. Candidates in this category should not consider themselves to be at a disadvantage because their Training Record will appear to be incomplete. New trainees will be advised when this special provision will no longer apply.

\section{SECTION 2 \\ Curriculum for Higher Specialist Training in Accident and Emergency Medicine}

\section{1 \\ Overview of Accident and Emergency Medicine}

The orientation of training in Accident and Emergency Medicine shall encompass the following:

\subsubsection{PRINCIPLES OF EMERGENCY CARE}

(1) Organisational issues and quality standards

(2) Manpower and skill mix

(3) Resuscitation, recognition of threats to life and limb

(4) Triage of the emergency department patient

(5) Understanding of "timelines" and documentation

(6) Interface with primary/community care

(7) Therapeutics and pain control

(8) Patient dignity and privacy

(9) Ethical issues and confidentiality

\subsubsection{EMERGENCY MEDICAL SERVICES}

(1) Pre-hospital care and the ambulance service

(2) Paramedic training and function

(3) Major Incident planning/procedures/practice

\subsubsection{EPIDEMIOLOGY OF ACCIDENTS AND EMERGENCIES}

2.1.4 ACCIDENT PREVENTION AND HEALTH PROMOTION Training is based on the acquisition of knowledge, skills, and attitudes.

\subsection{Knowledge base}

2.2.1 CARDIOVASCULAR DISEASES

A Cardiopulmonary resuscitation

(1) One and two rescuer CPR

(2) Choking victim

(3) Infant CPR

B Advanced cardiac life support (certification is expected)

(1) Treatment of ventricular fibrillation/ventricular tachycardia/asystole/electromechanical dissociation/bradyarrhythmias

(2) Protocols drugs and pacing

(3) Resuscitation team leadership

C Chest pain

(1) Ischaemic heart disease

(2) Myocardial infarction and thrombolysis

(3) Angina pectoris

(4) Pulmonary embolism

(5) Aortic dissection

D Heart failure and pulmonary oedema

E Supraventricular and ventricular tachycardias

F Hypertensive emergencies

G Cardiac pacemaker function/failure

H Peripheral vascular disease 
2.2.2 TRAUMA - RECOGNITION AND RESUSCITATION ROOM MANAGEMENT

A Advanced trauma life support (certification is expected)

(1) Primary survey/resuscitation

(2) Secondary survey

(3) Definitive care

(4) Transfer arrangements

B Head and facial trauma

C Chest and cardiac trauma

(1) Blunt/penetrating

(2) Tension pneumothorax

(3) Cardiac tamponade

(4) Massive haemothorax

(5) Open chest wound

(6) Ruptured aorta

(7) Blast injury

(8) Flail chest/lung contusion

D Abdominal trauma

(1) Blunt/penetrating

(2) Special investigations

E Genito-urinary trauma

F Extremity trauma

(1) Hand injuries - accurate diagnosis of bony, tendon and nerve injuries

(2) Skeletal trauma

(3) Vascular trauma

(4) Soft tissue injury

G Spinal trauma

(1) Immobilisation/logrolling techniques

H Paediatric trauma

I Trauma in pregnancy

2.2.3 ANAESTHESIA

A Principles of airway management

B Rapid sequence induction

C Pain relief

D General, regional, local anaesthesia

E Interface with Intensive Care

2.2.4 SHOCK

A Clinical findings and differential diagnosis

B Fluid resuscitation

C Blood/blood products

2.2.5 PULMONARY EMERGENCIES

A Acute respiratory failure

B Asthma

C Chronic obstructive airways disease

D Foreign body

E Pneumothorax

F Chest infection

G Thermal/chemical injury

$\mathrm{H}$ Evaluation of dyspnoea

I Poisoning

\subsubsection{GASTROINTESTINAL CONDITIONS}

A Acute GI bleed

B Vomiting, diarrhoea, and dehydration

C Abdominal pain evaluation

D Foreign body ingestion

2.2.7 OPHTHALMOLOGY

A The red eye

(1) Foreign body/corneal abrasion

(2) Conjunctivitis

(3) Acute glaucoma
B

Causes of visual impairment

D Orbital cellulitis

2.2.8 METABOLIC EMERGENCIES

A Hypoglycaemia

B Ketoacidosis/hyperosmolar coma

C Electrolyte abnormalities

D Acid-base abnormalities

2.2.9 GYNAECOLOGY AND OBSTETRICS

A Urinary tract infections

B Pelvic inflammatory disease/lower abdominal pain

C Ectopic pregnancy

D Antenatal bleeding

E Emergency delivery

2.2.10 GENITOURINARY MEDICINE

A Sexually transmitted diseases

B Epididymitis

C Testicular torsion

D Sexual assault

E Nephrolithiasis

2.2.11 INFECTIOUS DISEASES

A Hepatitis

B HIVIAIDS

C Tuberculosis

D Malaria

E Childhood illnesses

F Pyrexia of undetermined origin

2.2.12 TOXICOLOGY EMERGENCIES

A Recognition of clinical syndromes

B Initial treatment of poisoning

C Agent-specific therapy

D Role of poison centres

E Decontamination facilities/procedures (including radioactivity)

2.2.13 CHILDHOOD EMERGENCIES

A Advanced paediatric life support (certification is expected)

B Developmental paediatrics

C Croup/epiglottitis

D Asthma

E Fever

F Dehydration/gastrointestinal disorders

G Meningitis/septicaemia

$\mathrm{H}$ Seizures

I Congenital/inherited illness

$\mathrm{J} \quad$ Pain relief

$\mathrm{K} \quad$ Parasuicide in adolescents

L Child abuse

2.2.14 ENVIRONMENTAL EMERGENCIES

A Burns

(1) Grading/resuscitation

(2) Smoke inhalation

(3) Outpatient treatment

B Heat illness

C Hypothermia

D Near drowning

E Electrical injury (including lightning) 
F Bites

(1) Snake/dog/cat/human/arthropod

(2) Rabies/tetanus prophylaxis

G Anaphylaxis

\subsubsection{ENT CONDITIONS}

A Epistaxis/septal haematoma

B Foreign bodies

C Infections

D Upper airway obstruction

E Dental emergencies

2.2.16 NEUROLOGICAL EMERGENCIES

A Coma

B Headache/subarachnoid haemorrhage

C Meningitis

D Seizures

E Stroke

2.2.17 MUSCULOSKELETAL CONDITIONS

A Orthopaedic and neurovascular extremity examination

B Strains/sprains/fractures

C Dislocations

D Septic joint

E Soft tissue injury/infection

F Nerve entrapment syndromes

$G \quad$ Back pain

$\mathrm{H} \quad$ Locked joint

I Rheumatological disorders

\subsubsection{BEHAVIOURAL EMERGENCIES}

A Mental state examination

B Organic illness manifest as behavioural disorders

C Acute psychosis

D Deliberate self harm

E Suicidal and homicidal evaluation

F Alcohol misuse

\subsubsection{GERIATRIC EMERGENCIES}
A Psychosocial assessment
B Mental state examination
C Mobility assessment
D Multi-system pathology

\subsubsection{SOCIAL EMERGENCIES}
B Frequent attenders
C Homelessness
D Alcohol related problems
E Hospital hopper
F Drug misuse
A Overall care of the patient

2.2.21 HAEMATOLOGICAL EMERGENCIES

A Haemoglobinopathies

B Haemophilia

2.2.22 LEGISLATION/STATUTORY CIRCULARS

$\begin{array}{ll}\text { A } & \text { Common Law and confidentiality } \\ \text { B } & \text { Children Act } \\ \text { C } & \text { Mental Health Act } \\ \text { D } & \text { Health and Safety at Work Act } \\ \text { E } & \text { Road Traffic Act } \\ \text { F } & \text { Data Protection Act } \\ \text { G } & \text { Access to Health Records Act } \\ \text { H } & \text { Patients' Charter } \\ \text { I } & \text { NHS and Community Care Act }\end{array}$

$\mathrm{J} \quad$ Role of the Coroner

K Organ/tissue donation

L Hospital Complaint Procedure Act

M Equal opportunities

\subsection{Clinical skills}

2.3.1 WOUND MANAGEMENT

A Types of wounds/extremity examination

B Wound preparation

C Wound closure techniques

D Foreign bodies

E Extensor tendon repair

F Incision of abscesses and pulp space infections

G Flexor tendon sheath infections (including palmar spaces)

$\mathrm{H} \quad$ Finger tip injuries

I Dressing techniques

J Plaster techniques

$\mathrm{K}$ Joint aspiration, soft tissue injection

L Anaesthetic techniques

2.3.2 CARDIOPULMONARY RESUSCITATION AND MEGACODE TRAINING (ie, ACLS)

A Cardioversion/carotid sinus massage

B Emergency thoracotomy

C Breaking bad news

\subsubsection{MAJOR TRAUMA MANAGEMENT (ie, ATLS) AND} TEAM LEADER FUNCTION

2.3.4 AIRWAY (CERVICAL SPINE CONTROL)

A Basic airway management

B Advanced airway management (tracheal intubation/alternatives)

C Surgical airway

2.3.5 BREATHING

A Needle/tube thoracotomy

B Ventilation techniques

2.3.6 CIRCULATION

A Central venous access

B Arterial access

C Cut down techniques

D Femoral vessel cannulation

E Pericardiocentesis

F Intra-osseous access

G CVP monitoring

\subsubsection{DIAGNOSTIC PERITONEAL LAVAGE}

2.3.8 SPLINTING/IMMOBILISATION

A Spinal immobilisation

B Limb splinting/traction splints

C Logrolling

2.3.9 ENT

A Indirect laryngoscopy

B Nasal packing

2.3.10 MAXILLO-FACIAL

A Dental anaesthesia

B Dental socket suture

C Plastic surgery techniques 
2.3.11 REDUCTION OF FRACTURES/DISLOCATIONS (LOCAL AND REGIONAL ANAESTHETIC TECHNIQUES/PAIN RELIEF)

2.3.12 GASTRIC LAVAGE

2.3.13 SLIT LAMP

2.3.14 EMERGENCY DELIVERY

2.3.15 INTERPRETATION OF RADIOGRAPHS (CT/MRI INCLUDED)

2.3.16 TRANSPORTATION OF PATIENTS

\subsection{Communication skills}

2.4.1 PROFESSIONAL RELATIONSHIPS

A Patients and relatives

B Colleagues

C Other staff

2.4.2 BEREAVEMENT CARE

\section{$2.5 \quad$ Managerial skills}

2.5.1 DEPARTMENT POLICIES/PROCEDURES

2.5.2 STAFF MANAGEMENT (MANPOWER/PERSONNEL PROCEDURES)

2.5.3 EQUIPMENT (CHOOSING TO ORDERING)

2.5.4 RESOURCE MANAGEMENT/CLINICAL BUDGETING

2.5.5 CONTRACTING/STANDARDS SETTING

2.5.6 INFORMATION TECHNOLOGY

2.5.7 CLINICAL AUDIT/QUALITY MONITORING

2.5.8 COMPLIMENTS/COMPLAINTS

2.5.9 MEDICOLEGAL STATEMENTS
2.5.10 COMMITTEE WORK

2.5.11 LIAISING WITH OTHER AGENCIES

2.5.12 PUBLIC RELATIONS/MEDIA

2.5.13 MAJOR INCIDENT PLANNING/EXERCISES

\section{$2.6 \quad$ Teaching skills}

2.6.1 LECTURE PREPARATION

2.6.2 SMALL GROUP TECHNIQUES

2.6.3 PRESENTATION TECHNIQUES

2.6.4 TEACHING CRITIQUE

2.6.5 DEPARTMENTAL TEACHING PROGRAMME

2.6.6 PROFESSIONAL DEVELOPMENT (SEIF DIRECTED LEARNING)

\section{$2.7 \quad$ Research skills}

2.7.1 LITERATURE SURVEY

2.7.2 SCIENTIFIC STUDY DESIGN

2.7.3 DATA EVALUATION/STATISTICS

2.7.4 PREPARING PUBLICATIONS

\section{$2.8 \quad$ Attitudes}

2.8.1 LEADERSHIP

2.8.2 RELIABILITY

2.8.3 TEAMWORK

2.8.4 SELF MOTIVATION 


\section{SECTION 3}

\section{Training Record for Higher Specialist Trainees in Accident and Emergency Medicine}

\subsection{Introduction}

The aim of this training record is to provide a document which gives details of your time and experience in higher specialist training in Accident and Emergency Medicine. It also provides a structure for your annual appraisal and the formulation of your training programme with your trainer.

You should begin to fill in your record as soon as you take up your training post. Your initial training plan should be agreed with your trainer in the first month of your post. It will then be reviewed at your six month appraisal and annually thereafter. You should keep this record up to date - entries should be made as and when the relevant experience is gained.

At the annual appraisals your trainer will review your general progress and the skills sections with you. Each skill

\subsection{Training Record Proforma}

will be signed up only when your trainer is satisfied that you are competent in that skill to an acceptable standard for independent practice. It is recognised that not every trainee will necessarily have the opportunity to perform each and every skill.

The skills sections are not meant to be comprehensive but have been issued as guidance by the Specialist Advisory Committee and the Faculty of Accident and Emergency Medicine as part of the curriculum for higher specialist training. The knowledge base of the curriculum will be tested by the Specialty Examination in Accident and Emergency Medicine (FFAEM).

Your training record should be presented to the examiners as part of the Specialty Examination and it will also be reviewed as part of the final decision on certification. 


\title{
THE FACULTY OF ACCIDENT AND EMERGENCY MEDICINE
}

\author{
TRAINING RECORD \\ FOR \\ HIGHER SPECIALIST TRAINEES \\ IN \\ ACCIDENT AND EMERGENCY \\ MEDICINE
}




\section{SECTION 1 - SUMMARY OF TRAINING}

\section{Posts Prior to A \& E Higher Specialist Training}

Document in chronological order your professional experience prior to the commencement of your higher specialist training post.

\begin{tabular}{|l|l|l|l|l|}
\hline Grade & Specialty & Hospital & Dates & FT/PT \\
\hline & & & & \\
& & & & \\
& & & & \\
\hline
\end{tabular}

\section{Qualifications}

\begin{tabular}{|l|l|l|}
\hline Qualification & Date awarded & Institution \\
\hline & & \\
& & \\
\hline
\end{tabular}

\section{Summary of A \& E Higher Specialist Training}

This is a chronological summary of all Accident and Emergency posts since starting your higher specialist training. It should be regularly updated.

\begin{tabular}{|l|l|l|l|}
\hline Hospital & Trainer/s & Dates & FT/PT \\
\hline & & & \\
& & & \\
& & & \\
\hline
\end{tabular}

\section{Secondments}

Essential secondments should be full time or the equivalent. Others may be part-time in which case the amount of commitment per week should be specified.

\begin{tabular}{|l|l|l|l|l|}
\hline Specialty & Hospital & Supervisor/s & Dates & FT/PT \\
\hline & & & & \\
& & & & \\
& & & & \\
\hline
\end{tabular}




\section{SECTION 2 - SKILLS}

\section{Practical}

This section lists a core of practical skills that the trainee should learn. Although they will be reviewed annually, your trainer will sign the entry as and when satisfied that you are competent to perform the skill to a standard acceptable for independent practice.

\begin{tabular}{|c|c|c|c|c|c|c|c|c|c|c|}
\hline & \multicolumn{2}{|c|}{ Year 1} & \multicolumn{2}{|c|}{ Year 2} & \multicolumn{2}{|c|}{ Year 3} & \multicolumn{2}{|c|}{ Year 4} & \multicolumn{2}{|c|}{ Year 5} \\
\hline & Date & Initials & Date & Initials & Date & Initials & Date & Initials & Date & Initials \\
\hline Resuscitation Skills: & & & & & & & & & & \\
\hline Basic life support & & & & & & & & & & \\
\hline Defibrillation & & & & & & & & & & \\
\hline Cardioversion & & & & & & & & & & \\
\hline $\begin{array}{l}\text { Advanced Airway } \\
\text { Management }\end{array}$ & & & & & & & & & & \\
\hline Ventilation with BVM system & & & & & & & & & & \\
\hline Endotracheal intubation & & & & & & & & & & \\
\hline $\begin{array}{l}\text { Surgical airway } \\
\text { (cricothyroidotomy) }\end{array}$ & & & & & & & & & & \\
\hline $\begin{array}{l}\text { Ventilation of intubated } \\
\text { patients }\end{array}$ & & & & & & & & & & \\
\hline $\begin{array}{l}\text { Use of paralysing and other } \\
\text { anaesthetic drugs }\end{array}$ & & & & & & & & & & \\
\hline Vascular Access: & & & & & & & & & & \\
\hline Central lines & & & & & & & & & & \\
\hline CVP monitoring & & & & & & & & & & \\
\hline IV cutdown techniques & & & & & & & & & & \\
\hline Femoral vein lines & & & & & & & & & & \\
\hline Intraosseous infusion & & & & & & & & & & \\
\hline Arterial line insertion & & & & & & & & & & \\
\hline Other Skills: & & & & & & & & & & \\
\hline $\begin{array}{l}\text { Team leadership of } \\
\text { cardiac/trauma resuscitation }\end{array}$ & & & & & & & & & & \\
\hline Pericardiocentesis & & & & & & & & & & \\
\hline Peritoneal lavage & & & & & & & & & & \\
\hline $\begin{array}{l}\text { Chest drain } \\
\text { insertion/thoracocentesis }\end{array}$ & & & & & & & & & & \\
\hline Emergency thoracotomy & & & & & & & & & & \\
\hline $\begin{array}{l}\text { Spinal immobilisation } \\
\text { techniques/log rolling }\end{array}$ & & & & & & & & & & \\
\hline $\begin{array}{l}\text { Transportation of seriously } \\
\text { ill patients }\end{array}$ & & & & & & & & & & \\
\hline Bereavement care & & & & & & & & & & \\
\hline Fractures and Dislocations: & & & & & & & & & & \\
\hline $\begin{array}{l}\text { Closed fracture manipulation } \\
\text { (eg Colles, fingers) }\end{array}$ & & & & & & & & & & \\
\hline $\begin{array}{l}\text { Reduction of joint dislocation } \\
\text { (eg shoulders, ankles, fingers) }\end{array}$ & & & & & & & & & & \\
\hline $\begin{array}{l}\text { Joint and fracture } \\
\text { immobilisation techniques }\end{array}$ & & & & & & & & & & \\
\hline
\end{tabular}




\section{Practical (continued)}

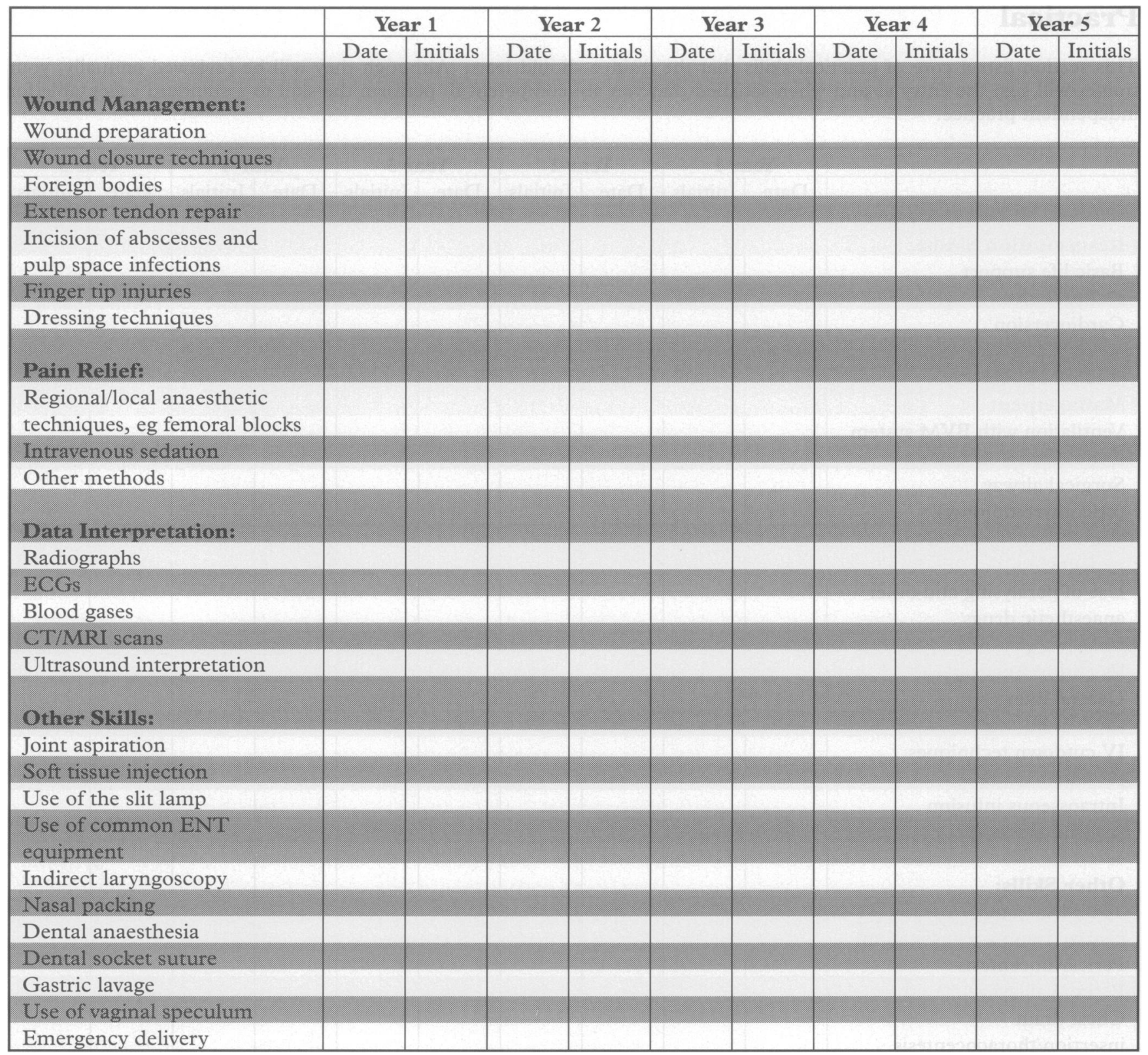




\section{Teaching}

\begin{tabular}{|c|c|c|c|c|c|c|c|c|c|c|}
\hline 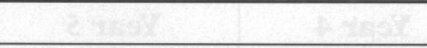 & \multicolumn{2}{|c|}{ Year 1} & \multicolumn{2}{|c|}{ Year 2} & \multicolumn{2}{|c|}{ Year 3} & \multicolumn{2}{|c|}{ Year 4} & \multicolumn{2}{|c|}{ Year 5} \\
\hline & Date & Initials & Date & Initials & Date & Initials & Date & Initials & Date & Initials \\
\hline Lecture preparation & & & & & & & & & & \\
\hline Presentation techniques & & & & & & & & & & \\
\hline Small group techniques & & & & & & & & & & \\
\hline Teaching critique & & & & & & & & & & \\
\hline $\begin{array}{l}\text { Organisation of departmenta } \\
\text { teaching programme }\end{array}$ & & & & & & & & & & \\
\hline $\begin{array}{l}\text { Professional development } \\
\text { (self directed learning) }\end{array}$ & & & & & & & & & & \\
\hline Teaching of: & & & & & & & & & & \\
\hline medical undergraduates & & & & & & & & & & \\
\hline medical postgraduates & & & & & & & & & & \\
\hline nursing staff & & & & & & & & & & \\
\hline paramedics & & & & & & & & & & \\
\hline other health professionals & & & & & & & & & & \\
\hline lay people & & & & & & & & & & \\
\hline
\end{tabular}

\section{Research}

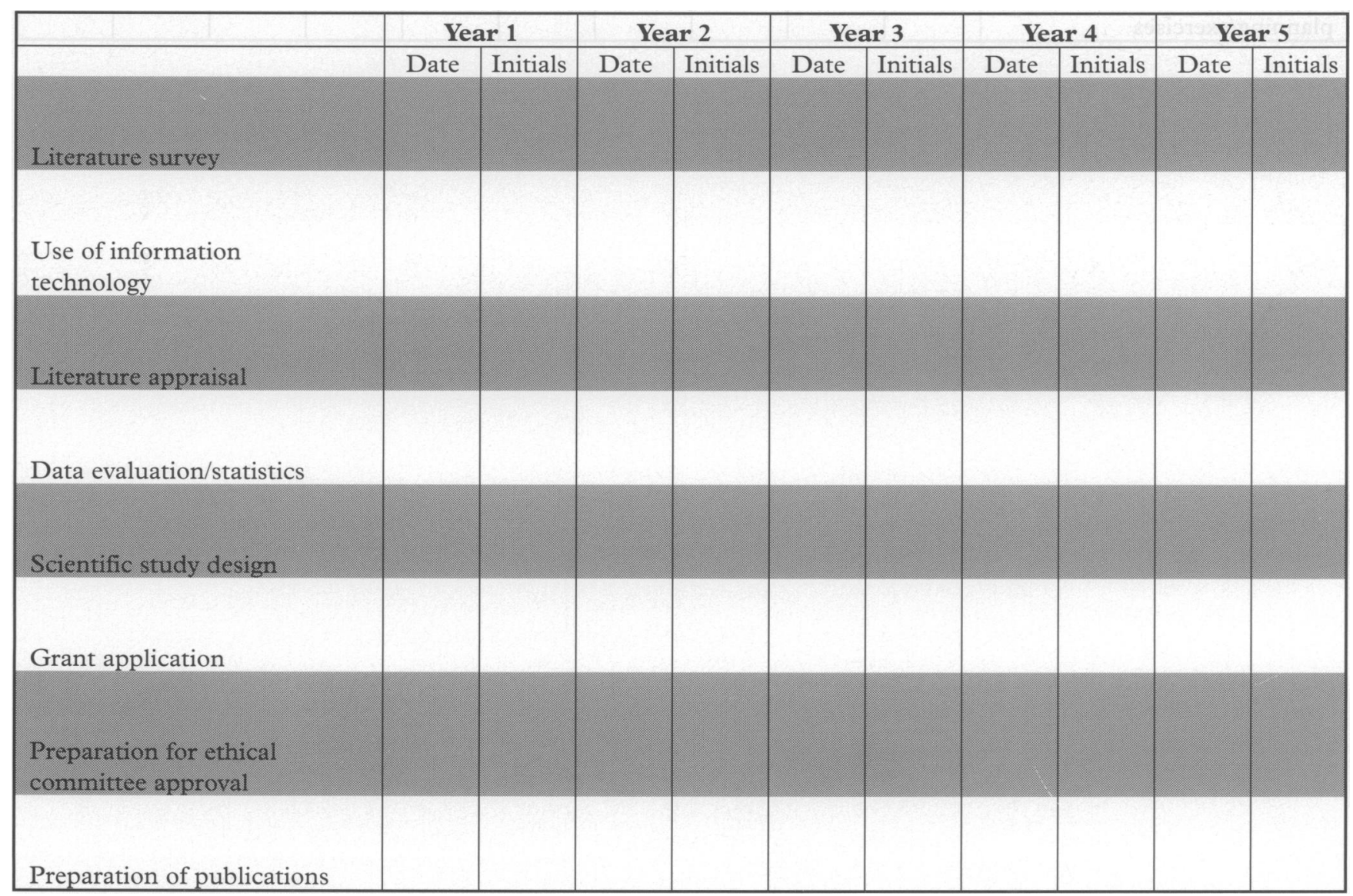




\section{Management}

\begin{tabular}{|c|c|c|c|c|c|c|c|c|c|c|}
\hline$+2 x^{2}=$ & \multicolumn{2}{|c|}{ Year 1} & \multicolumn{2}{|c|}{ Year 2} & \multicolumn{2}{|c|}{ Year 3} & \multicolumn{2}{|c|}{ Year 4} & \multicolumn{2}{|c|}{ Year 5} \\
\hline & Date & Initials & Date & Initials & Date & Initials & Date & Initials & Date & Initials \\
\hline $\begin{array}{l}\text { Departmental procedures and } \\
\text { policy making }\end{array}$ & & & & & & & & & & \\
\hline Staff management: & & & & & & & & & & \\
\hline recruiting & & & & & & & & & & \\
\hline disciplining & & & & & & & & & & \\
\hline appraisal & & & & & & & & & & \\
\hline day to day & & & & & & & & & & \\
\hline manpower issues & & & & & & & & & & \\
\hline Choosing/ordering equipment & & & & & & & & & & \\
\hline $\begin{array}{l}\text { Resource management and } \\
\text { clinical budgeting }\end{array}$ & & & & & & & & & & \\
\hline $\begin{array}{l}\text { Contracting and standard } \\
\text { setting }\end{array}$ & & & & & & & & & & \\
\hline Use of information technology & & & & & & & & & & \\
\hline Clinical audit: & & & & & & & & & & \\
\hline selection & & & & & & & & & & \\
\hline methodology & & & & & & & & & & \\
\hline use of data & & & & & & & & & & \\
\hline Quality monitoring & & & & & & & & & & \\
\hline $\begin{array}{l}\text { Dealing with } \\
\text { complaints/compliments }\end{array}$ & & & & & & & & & & \\
\hline $\begin{array}{l}\text { Medico-legal work/court } \\
\text { appearances }\end{array}$ & & & & & & & & & & \\
\hline $\begin{array}{l}\text { appearances } \\
\text { Committee work }\end{array}$ & & & & & & & & & & \\
\hline Liaison with other agencies & & & & & & & & & & \\
\hline Meeting with the media/PR & & & & & & & & & & \\
\hline Major incident & & & & & & & & & & \\
\hline
\end{tabular}




\section{SECTION 3 - OTHER EXPERIENCE}

\section{Pre-hospital Care}

\begin{tabular}{|l|l|l|}
\hline Experience & Place & Date/s \\
\hline & & \\
& & \\
& & \\
\hline
\end{tabular}

\section{Advanced Life Support Courses}

\begin{tabular}{|l|l|l|l|}
\hline & Date & Provider/Instructor & Location \\
\hline ATLS Recertification & & & \\
\hline ACLS/ALS Recertification & & & \\
\hline APLS/PALS Recertification & & & \\
\hline
\end{tabular}

\section{Other Courses, Conferences and Meetings}

Trainees may wish to include relevant experiences prior to Higher Specialist Training.

\begin{tabular}{|l|l|l|}
\hline Course/Conference/Meeting & Place & Date/s \\
\hline & & \\
& & \\
\hline
\end{tabular}

\section{Presentations}

Presentations to meetings outside the local Accident and Emergency Department should be listed here.

\begin{tabular}{|l|l|l|}
\hline Course/Conference/Meeting & Place & Date/s \\
\hline & & \\
& & \\
\hline
\end{tabular}




\section{Publications}

Please indicate title, author(s), journal reference in this order.

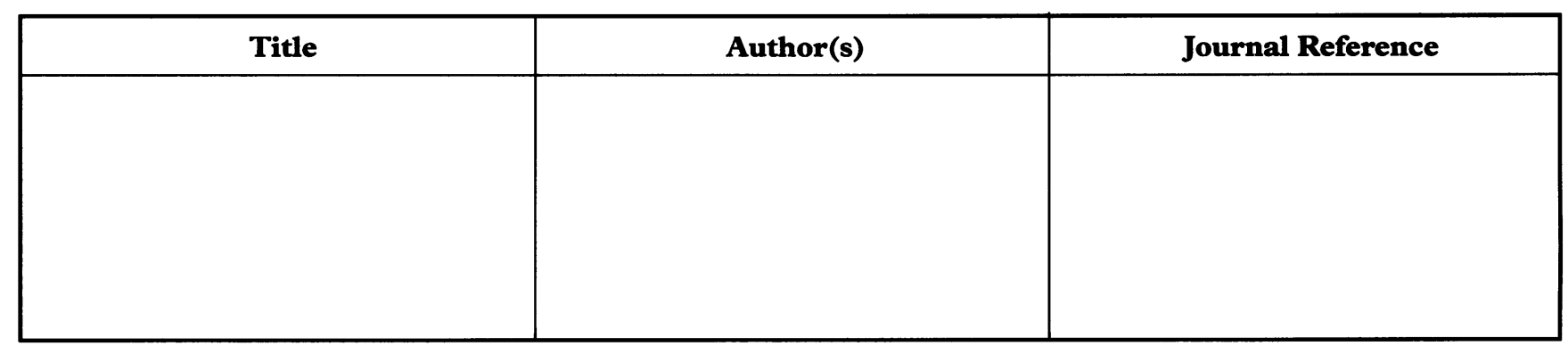

\section{Research Undertaken}

Give a brief summary of the objectives, methodology and results (maximum of 5 lines per project).

\subsection{Addendum}

Candidates who have completed periods of training before receiving the Regulations for the Examination and before receiving the Training Record will not be expected to complete their Training Record for such periods.

Candidates in this category should not consider themselves to be at a disadvantage because their Training Record will appear to be incomplete.

Trainees will be advised when this special provision will no longer apply. 